\section{Prescribing by Pharmacist's and Collaborative Care: Are We Ready to Accept the Baton and Get in the Race?}

We recently had the misfortune to come across the adverting campaign released by the Ontario Medical Association (OMA) that suggested, with some clever rhetoric, that physicians should be the only health care providers to prescribe drugs. ${ }^{1}$ This advertisement was accompanied by a picture of a presumed patient with hands raised in front of his or her face, as if the person was to be the next victim in a teenage slasher movie. It is quite simply a new iteration of an old argument: there is no evidence that prescribing by pharmacists benefits patients. However, does the absence of evidence equate with the absence of benefit? Or is it simply that we have never measured outcomes? Pharmacists now have the opportunity to obtain additional prescribing authority in Alberta, which permits initial-access prescribing and prescribing in the management of chronic diseases, based on independent assessments of patients or the referral of patients from other health care professionals. ${ }^{2.3}$ But are hospital pharmacists ready?

The OMA media campaign included the following statement: "Do [pharmacists] have the training in diagnosis and examination to [prescribe medications] properly?" In attempting to answer this question, one striking notion comes to mind: Why are pharmacists not considered an indispensable member of the health care team? We acknowledge that some teams rely heavily on their respective clinical pharmacists, but we all know that when we go home, the team functions adequately in our absence. Why aren't we expected to be available and to contribute like other health care professionals? While there may be times when we can contribute effectively to patient care in a retrospective manner, should this be the normal mode of contribution? The OMA has thrown down the gauntlet. It is now time for us to look beyond the current practice model and identify how we can further evolve.

To use an athletic analogy, the hospital-based health care team is not unlike a track and field relay team, in which each member is essential in order to finish race. Physicians have traditionally held the baton, running each leg of the race without assistance from others. The collaborative-care model encourages physicians to pass the baton to other qualified team members (e.g., nurses, physiotherapists), but pharmacists are often excluded. The best way to describe many of our practices is to say we run alongside physicians, recommending that they run faster! It's now time to stop cheering from the sidelines and get into the race. The collaborative practice model enables us to become more involved, such that physicians make the diagnosis and treatment decision, and pharmacists assume responsibility for managing the patient's drug therapy. ${ }^{4}$ Here are some basic principles to help pharmacy practitioners accept the baton and run their leg of the race. ${ }^{4}$

First, we are never going to be considered indispensable if we are not available and present in patient care areas. Making ourselves available will include abandoning the traditional "banker's hours" that many clinical pharmacists currently enjoy.
Pharmacists must find a way to be accessible when decisions for drug therapy are made, whenever that occurs.

Second, it is insufficient for us to remain just drug experts. In order to prescribe medications safely and effectively, we must utilize all information available to us. Therefore, we must extend our knowledge base to other areas and disciplines.

Third, we have traditionally preferred to leave the up-closeand-personal patient care to other members of the team. Moving forward requires us to get a little closer to the patient. By definition, "clinical pharmacists are involved in direct interaction with, and observation of, the patient" [emphasis added]. Basic physical examination skills support clinical decision-making and enable pharmacists to assume responsibility for managing patients' drug therapy.

Fourth, pharmacists must be prepared to develop their practice niche. Select one area to focus on, remembering that the lowest-hanging fruit is always easiest to pick. Start by providing the clinical pharmacy services that have been shown to reduce mortality rates. ${ }^{6}$ Initially, our expanded roles may be met with confusion and territorial struggles, but it is important to then perform these roles consistently and well. Don't expect the change to occur overnight, but persevere and focus on demonstrating your contributions to improved patient care. Document it! Measure it! Perhaps even publish it! Your efforts could help demonstrate the valuable role of pharmacists and inform a broad audience.

It is important that we take responsibility for our patients. We cannot allow organizations like the OMA to diminish our contributions to patient care. We believe that patients and physicians alike will grant us more credibility when we demonstrate competency and assume accountability for patient care. Like well-trained athletes, we must be ready to run our leg of the race when the baton is passed.

References

1. Ontario Medical Association, Section of General and Family Practitioners. With today's healthcare, is an apple a day your safest bet? Toronto $(\mathrm{ON})$ : Ontario Medical Association; 2009 [cited 2009 Oct 7]. Available from: www.familydoctorsofontario.ca

2. Pearson GJ. Evolution in the practice of pharmacy - not a revolution. CMAJ 2007;176(9):1295-1296.

3. What is pharmacist prescribing? Edmonton $(\mathrm{AB})$ : Alberta College of Pharmacists; 2009 [cited 5 Oct 2009]. Available from: https:// pharmacists.ab.ca/nPublic/PharmacistPrescribing.aspx

4. Barry AR, Pearson GJ. Making hospital pharmacists indispensable on the healthcare team: revolution in waiting. J Pharm Pract Res 2009;39(4): 256-257.

5. American College of Clinical Pharmacy. The definition of clinical pharmacy. Pharmacotherapy 2008;28(6):816-817.

6. Bond CA, Raehl CL. Clinical pharmacy services, pharmacy staffing, and hospital mortality rates. Pharmacotherapy 2007;27(4):481-493.

Arden R Barry, BSC, BSc(Pharm), ACPR

Faculty of Pharmaceutical Sciences

University of British Columbia

Vancouver, British Columbia

Glen J Pearson, BSC, BScPhm, PharmD, FCSHP

Division of Cardiology

Walter C Mackenzie Health Sciences Centre

University of Alberta

Edmonton, Alberta 